\title{
On the Tail of the Overlap Probability Distribution in the Sherrington-Kirkpatrick Model
}

\author{
Alain Billoire \\ Service de Physique Théorique, CEA/DSM/SPhT \\ Laboratoire associé au CNRS \\ CEA-Saclay, 91191 Gif-sur-Yvette cédex (France) \\ Silvio Franz \\ ICTP, Strada Costiera 11, PO Box 563 \\ 34100 Trieste (Italy) \\ Enzo Marinari \\ Dipartimento di Fisica, SMC and UdR1 of INFM and INFN \\ Università di Roma La Sapienza \\ P. A. Moro 2, 00185 Roma (Italy) \\ billoir@spht.saclay.cea.fr \\ franz@ictp.trieste.it enzo.marinari@roma1.infn.it
}

November 4, 2018

\begin{abstract}
We investigate the large deviation behavior of the overlap probability density in the Sherrington-Kirkpatrick model using the coupled replica scheme, and compare with the results of a large scale numerical simulation. In the spin glass phase we show that generically, for any model with continuous $\mathrm{RSB}, 1 / N \log P_{N}(q) \approx-\mathcal{A}\left(\left(|q|-q_{E A}\right)^{3}\right.$, and compute the first correction to the expansion of $\mathcal{A}$ in powers of
\end{abstract}


$T_{c}-T$ for the SK model. We also study the paramagnetic phase, where results are obtained in the replica symmetric scheme that do not involve an expansion in powers of $q-q_{E A}$ or $T_{c}-T$. We give finally precise semi-analytical estimates of $P(|q|=1)$. The overall agreement between the various points of view is very satisfactory.

\section{Introduction}

Although the solution of the Sherrington-Kirkpatrick (SK) model was proposed more than two decades ago [1], and it physical interpretation is (now) quite clear [2], some fundamental aspects of the model are still poorly understood. We are thinking here about the problem of the time scales of the model [3], and to the question of chaos with respect to temperature variations [4, 5, just to quote some recent works.

In this article, we will revisit the question of the large deviation behavior of the order parameter probability distribution $P_{N}(q)$ of the SK model [6, 7, 8], using new analytical results, much improved numerical data and refinements in the data analysis.

In the usual mean field approximation the probability distribution of the order parameter $P_{N}(m)$ is read from the free energy considered as a function of the order parameter. This method cannot be pursued in the replica approach, since the replica free energy has no physical interpretation for values of the order parameter different from the saddle point [2. The coupled replica method was proposed in 6] to circumvent this problem and used in [7, 8. In short, the replica method is applied to a system of two identical copies of the original system with a constrained value of the mutual overlap $q$, with the result:

$$
\frac{1}{N} \log P_{N}\left(|q|>q_{E A}\right)=-\mathcal{A}\left(|q|-q_{E A}\right)^{3}+\mathcal{O}\left(\left(|q|-q_{E A}\right)^{4}\right),
$$

where $q_{E A}$ is the Edwards-Anderson order parameter (the maximum allowed value for the overlap in the infinite volume limit). The proportionality coefficient $\mathcal{A}$ was computed in [6] to zeroth order in $\tau=\left(T_{c}^{2}-T^{2}\right) / 2 T_{c}$. In order to obtain a value of the coefficient $\mathcal{A}$ to compare with the results of our large scale simulation using the parallel tempering algorithm, we compute here the first order term in $\tau$. We also present semi-analytical results for $P(|q|=1)$. For $T \geq T_{c}$ we 
present the results of an exact calculation, made in the replica symmetric scheme, that is limited neither to small $q$ 's nor to the vicinity of $T_{c}$. This calculation corroborates nicely the other analytical and numerical results.

There are somehow two aspects in the comparison made between the analytical results and the Monte Carlo data. The first is to (cross) check both results. This is important since on one hand the analytical computation is very delicate and involves some unproven assumptions. The simulation is, on the other hand, limited to small systems finite number of disorder samples and finite statistics. Both approach are very complementary. The second aspect is in checking how much of the infinite volume physics (at low temperatures, in the spin glass phase) is already encoded in the sizes we can effectively study on our current state of the art computers (up to $N=4096$ in the present case): we will see that, as far as the present computation is concerned, things do work well, and we are moving on solid ground.

\section{Large Deviations in $P_{N}(q)$ for $|q|>$}

$q_{E A}$

In this section ${ }^{1}$ we sketch the main steps and give the results of our computations of the function $P_{N}(q)$ in the large deviation regime, i.e. for $|q|>q_{E A}$, for both $T<T_{c}$ and $T \geq T_{c}$.

The overlap probability distribution $P_{N, J}(q)$ depends on the disorder sample $J$, and the function $P_{N}(q)$ is obtained as $\overline{P_{N, J}(q)}$, where the over-bar denotes the disorder average. It is well known that in the thermodynamic limit the disorder average of $P_{N, J}(q)$ is non-vanishing ${ }^{2}$ in the interval $\left[0, q_{E A}\right]$, while sample to sample fluctuations remain strong even for large systems [2]. The probability of the complementary interval, i.e. of events with $q>q_{E A}$, is exponentially small in the system size [6]. For typical samples one has, independently of the disorder realization,

$$
P_{N, J}(q) \approx \tilde{P}_{N}(q) \propto \exp (-\beta N F(q)),
$$

where $\beta=1 / T$ and $F(q)$ is the self-averaging free energy cost of

\footnotetext{
${ }^{1}$ In the following we will take, without loss of generality, $q>0$ (assuming for example the presence of a suitable infinitesimal magnetic field).

${ }^{2}$ We assume that our starting Hamiltonian is symmetric under global spin reversal.
} 
keeping two replicas at a mutual overlap $q$ :

$$
F(q)=-\frac{T}{N} \overline{\log \left(\frac{1}{Z^{2}} \sum_{\left\{\sigma_{i}, \tau_{i}\right\}} e^{-\beta(H(\sigma)+H(\tau))}\left(q-\frac{1}{N} \sum_{i} \sigma_{i} \tau_{i}\right)\right)} .
$$

It is quite natural to expect that in the thermodynamic limit $P(q)=$ $\tilde{P}(q)$. In an alternative scenario the fluctuations of $P_{N, J}(q)$ are dominated by rare (i.e. having exponentially vanishing probability) samples, causing $\overline{P_{N, J}(q)}$ to be different from $\tilde{P}_{N}(q)$. We will discuss this issue with our numerical simulations in the next section.

Let us start with the case $T<T_{c}$. In reference [6] the computation of $F(q)$ has been performed using the method of coupled replicas in the glassy phase $\left(T<T_{c}\right)$ close to $T_{c}$, with the result that, in leading order in both $\tau \equiv\left(T_{c}^{2}-T^{2}\right) / 2 T_{c}$ and $q-q_{E A}$, on has $F=1 / 6\left(q-q_{E A}\right)^{3}$. Let us now perform the computation of the prefactor of the cubic term to the next order in $\tau$. In order to compute $F(q)$ one has to replicate $n$ times both the spins $\sigma_{i}$ and the spins $\tau_{i}$. Two order parameter matrices appear:

$$
Q_{a b}=\left\langle\sigma_{a} \sigma_{b}\right\rangle=\left\langle\tau_{a} \tau_{b}\right\rangle \text { and } P_{a b}=\left\langle\sigma_{a} \tau_{b}\right\rangle, a, b=1, \ldots, n .
$$

One makes a Parisi ansatz 2] for both $Q$ and $P$, introducing two functions $q(x)$ and $p(x)$. We now recast the two $n \times n$ matrices into a single $2 n \times 2 n$ bold-faced matrix

$$
\mathbf{Q}=\left(\begin{array}{ll}
Q & P \\
P & Q
\end{array}\right)
$$

Let us consider in all generality any system that is described in the un-coupled case by the free energy functional $F[Q]$ in terms of the usual replica matrix. The same system when coupled according to (2) has a free energy functional of the form

$$
F_{2}[Q, P]=F[\mathbf{Q}]+\epsilon\left(n q-\sum_{a=1}^{n} P_{a a}\right),
$$

where $\epsilon$ is a Lagrange multiplier associated to the $\delta$-function in (2).

If the problem of a single, un-coupled, system admits a continuous solution $q_{F}(x)$ (that we suppose to be known), it can be shown that the corresponding variational equations related to the coupled problem admit two simple solutions [9]. 
A first solution has $\Delta F=\epsilon=0$, with functions $q(x)$ and $p(x)$ that can be constructed explicitly from $q_{F}(x)$ :

$$
q(x)=\left\{\begin{array}{ll}
q_{F}(2 x) & 0 \leq x \leq \frac{\tilde{x}}{2} \\
q & \frac{\tilde{x}}{2} \leq x<\tilde{x} \\
q_{F}(x) & 1 \geq x \geq \tilde{x}
\end{array} \quad p(x)= \begin{cases}q_{F}(x) & 0 \leq x \leq \tilde{x} \\
q & \tilde{x} \leq x<1\end{cases}\right.
$$

where the point $\tilde{x}$ is defined by the equation $q_{F}(\tilde{x})=q$.

In the second solution $q(x)=p(x)$. This second solution becomes degenerate with the first one at $q=q_{E A}$. If we take the usual replica approach attitude to maximize the free energy with respect to the $Q$ parameters (in agreement with the physical intuition about the problem) we will select the first solution in the region with $q<q_{E A}$ and the second one in the region with $q>q_{E A}$, where it has a larger free energy.

Notice that while the first solution has a flat free energy $F(q)=0$, the second, as a consequence of the facts that

- it coincides with the first one for $q=q_{E A}$;

- it has to be a solution of the saddle point equations;

must be such that $\left.\frac{\mathrm{dF}(\mathrm{q})}{\mathrm{dq}}\right|_{q=q_{E A}}=0$. All these properties together imply that generically $F(q)=\mathcal{A}\left(q-q_{E A}\right)^{3}$, where $\mathcal{A}$ is a problem and temperature dependent constant. Of course the accidental vanishing of $\mathcal{A}$ is an allowed possibility, and in that case $F(q)$ would be of order five or higher.

Let us now turn to the explicit computation for the SK model close to $T_{c}$. In this case the replica free energy density can be written as

$$
F_{2}[Q, P]=\tau \operatorname{Tr} \mathbf{Q}^{* 2}+\frac{1}{3} \operatorname{Tr} \mathbf{Q}^{* 3}+\frac{1}{4} y \sum_{\alpha, \beta}^{1,2 n} \mathbf{Q}_{\alpha, \beta}^{*}{ }^{4}+\epsilon\left(n q-\sum_{a=1}^{n} P_{a a}^{*}\right),
$$

where $y=2 / 3$, but will be kept as a parameter during the computation, and we have defined $Q_{a b}^{*} \equiv Q_{a b} / T^{2} \simeq Q_{a b}(1+2 \tau)$ and $P_{a b}^{*} \equiv P_{a b} / T^{2}$. Here the solution can be found explicitly, writing $\tilde{p}^{*}=P_{a a}^{*}$ and inserting $P_{a b}^{*}=Q_{a b}^{*}(a \neq b)$ in the equation of motion one finds that the matrix $Q^{*}$ verifies the equation:

$$
\left(\tau+\tilde{p}^{*}\right) Q_{a b}^{*}+\left(Q^{* 2}\right)_{a b}+\frac{y}{2} Q_{a b}^{* 3}=0,
$$

which is identical to the solution of the free problem with $\tau \rightarrow\left(\tau+\tilde{p^{*}}\right) / 2$ and $y \rightarrow y / 2$. Plugging then the known solution of the free problem 
into the free energy functional it is easy to derive the result:

$$
F(q)=\left(\frac{1}{6}-\frac{3}{4} \tau y\right)\left(q^{*}-q_{E A}^{*}\right)^{3} .
$$

Or, using the original definition of $q$, and setting $y=2 / 3$,

$$
F(q)=\left(\frac{1}{6}+\frac{1}{2} \tau\right)\left(q-q_{E A}\right)^{3}
$$

Let us now discuss the paramagnetic phase, where $T>T_{c}$. Here $q_{E A}=0$ and we have to select the solution that has always $p(x)=q(x)$ for $q \geq 0$ and $p(x)=-q(x)$ for $q<0$. Let us consider the replica symmetric free energy, corresponding to $\tilde{p}=q, q(x)=\operatorname{sgn}(q), p(x)=$ $q_{0}$. For simplicity we will write as before equations valid for $q>0$, keeping in mind that the free energy $F(q)$ has to satisfy $F(-q)=F(q)$.

Defining $D y=\mathrm{e}^{-\frac{1}{2} y^{2}} / \sqrt{2 \pi} d y$, standard manipulations lead to the expression

$$
\begin{aligned}
F(q) & =-\frac{\beta}{2}\left(1+q^{2}+2 q_{0}^{2}-2 q_{0}-2 q q_{0}\right)-T \log 2+T \nu q \\
& -T \int D y \log \left[\mathrm{e}^{-\nu}+\mathrm{e}^{\nu} \cosh \left(2 \beta \sqrt{q_{0}} y\right)\right],
\end{aligned}
$$

that has to be extremized with respect to $q_{0}$ and $\nu$ (the Lagrange multiplier associated to $q$ ). This leads to the saddle point equations

$$
\begin{aligned}
q & =\int D y \frac{-\mathrm{e}^{-\nu}+\mathrm{e}^{\nu} \cosh \left(2 \beta \sqrt{q_{0}} y\right)}{\mathrm{e}^{-\nu}+\mathrm{e}^{\nu} \cosh \left(2 \beta \sqrt{q_{0}} y\right)} \\
q_{0} & =\frac{1+q}{2}-\frac{1}{2 \beta \sqrt{q_{0}} y} \int D y \frac{y \mathrm{e}^{\nu} \sinh \left(2 \beta \sqrt{q_{0}} y\right)}{\mathrm{e}^{-\nu}+\mathrm{e}^{\nu} \cosh \left(2 \beta \sqrt{q_{0}} y\right)} .
\end{aligned}
$$

These equations have always the trivial solution $q_{0}=0, \nu=\tanh ^{-1} q$, which is correct at high enough temperatures and small values of $q$. A small $q_{0}$ expansion reveals that a solution with $q_{0} \neq 0$ is possible for $q \geq T-1=q_{c}(T)$. Notice that

- for $T \leq 1$ the solution is non-trivial for all values of $q$,

- for $T>2$ the solution is always $q_{0}=0$,

- for $1<T \leq 2$ there is a phase transition from the solution $q_{0}=0$ for $q<T-1$ to a solution $q_{0} \neq 0$ for $q>T-1$. 


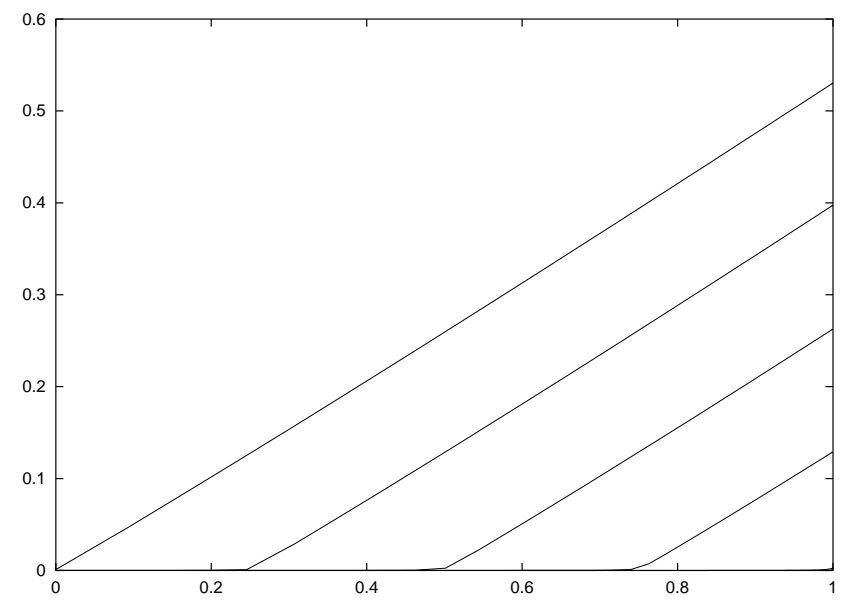

Figure 1: The induced overlap $q_{0}$ as a function of the mutual overlap $q$ that we force on the system for $T=1.0,1.25,1.5,1.75$ and 2.00 (from right to left, with $q_{0}=0 \forall q$ for $\left.T=1\right)$.

The correct solution in this last domain should break the replica symmetry. Close to $q_{c}(T)$ a small $q$ expansion as in the previous section can be used even for large values of $T_{c}-T$. For $q_{c}(T)-q \approx 1$ we only consider the replica symmetric approximation. It is clear that if we impose $q=1$ the system behaves as a single system with temperature $T / 2$, and we understand therefore why $q_{c}(2)=1$.

The $q_{0}=0$ solution gives a free energy

$$
F(q)=-\frac{\beta}{2}\left(1+q^{2}\right)-2 T \log 2+T \nu q-T \log (\cosh (\nu)) .
$$

In figure (11) we show the solution for $q_{0}$ as a function of $q$. For $q=1$ we find that $q_{0}$ takes, as it should, the value $q_{R S}(T / 2)$, i.e. the EdwardsAnderson value at temperature $T / 2$ in the $\mathrm{RS}$ approximation. The functional form of $q_{0}$ is well approximated for all values of $T$ by $q_{0}(q)=$ $q_{R S}(T / 2)\left(q-q_{c}(T)\right)$, although small quadratic deviation from this form are observable. In figure (2) we also plot the related free energy. 


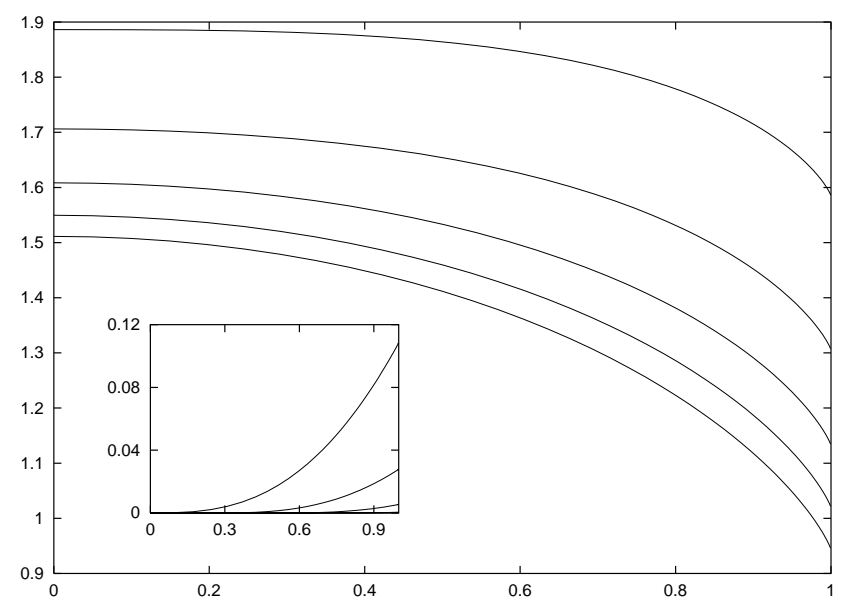

Figure 2: The free energy $-\beta F(q)$ for the same values of the temperature as in figure 1 (from top to bottom). In the insert we plot, for the same set of temperatures again, the difference $-\beta\left(F_{0}(q)-F(q)\right)$, where $F_{0}(q)$ is computed with the trivial solution $q_{0}=0$ (from top to bottom. The difference is zero for $T=2$ and very small for $T=1.75)$.

\section{$3 \quad$ Numerical versus Analytic Results}

In the following we will use our numerical results obtained from simulations of the $J_{i, j}= \pm 1$ SK model toward two different goals, that we have already partially discussed in the introduction. First we want to have a numerical check of our analytical findings: it is clear that we are dealing with complex set of equations, we are working in some asymptotic regimes, and this implies that a numerical check is quite welcome. The second issue is maybe less direct but also very crucial. It is important to understand how well numerical simulations done on finite lattice encompass the infinite volume physics, and, at the same time, how good a control we can have on the temperature range that we explore. The issue of the infinite volume limit is indeed of very large importance, and it is crucial to verify that exotic phenomena (like the large deviation regime we are discussing here) are already well quantified in the region of lengths we can study with nowadays computer facilities.

Our large scale numerical simulations are based on the numerical optimized Monte Carlo technique of parallel tempering [10]. They have already been used in our recent paper on the problem of chaos [5] (but 


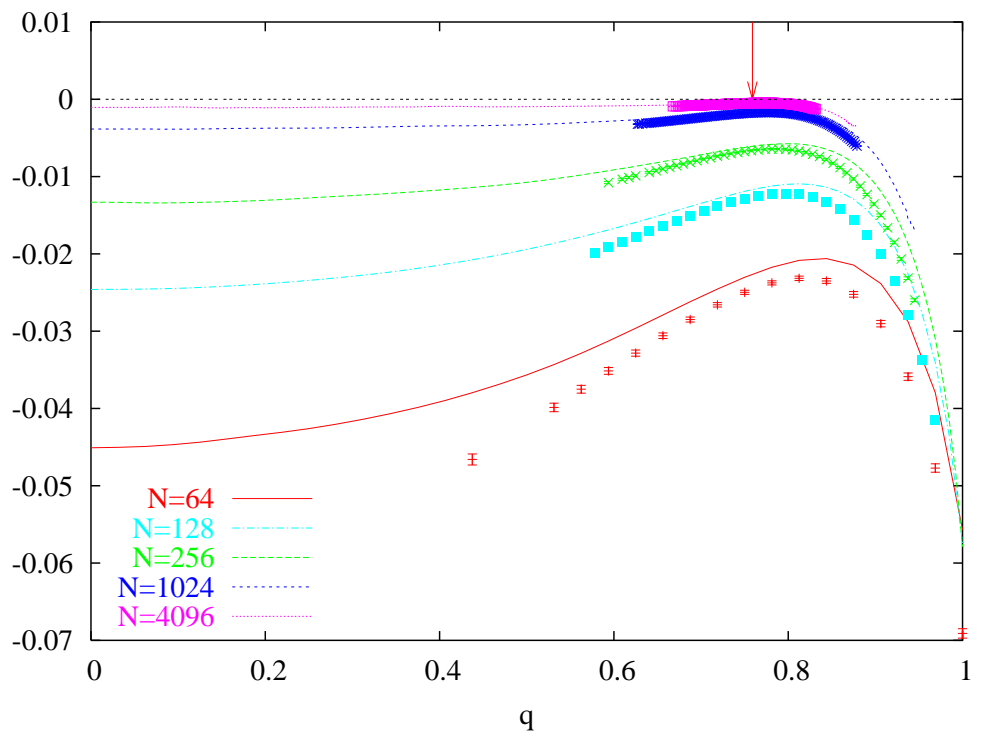

Figure 3: $\quad 1 / N \log \left(P_{N}(q) N^{-\frac{1}{3}}\right)$ versus $q$ for $T=0.4:$ annealed $(F(q)$, represented by lines) and quenched $(\tilde{F}(q)$, represented by points with error bars) estimates. The vertical arrow indicates the value of $q_{E A}$ at $T=0.4$.

for the addition of the data obtained on a $N=128$ site lattice).

We have analyzed 1024 different disorder realizations for $N=$ 64, 256 and 1024 sites, 256 for $N=4096$ and 8192 for $N=128$. We go down to a minimal temperature value $T_{m}=0.4 T_{c}=0.4$, and we use $0.410^{6}$ iterations for thermalizing and $10^{6}$ iterations for measurements. We normalize probability distributions according to $\int_{-1}^{1} P_{N}(q) d q=1$.

The first question we discuss is whether it is appropriate to measure the quenched average

$$
\beta \tilde{F}(q)=N^{-1} \overline{\log P_{N, J}(q)},
$$

(that is the quantity computed using the two-replica method) or if we can be happy by measuring the annealed average

$$
\beta F(q)=N^{-1} \log \overline{P_{N, J}(q)},
$$

(a quantity that is much easier to measure with Monte Carlo simulation). In section 2 we have discussed the issue, arguing that these 
two averages could differ because of rare samples. We now turn to our numerical data in order to answer this question.

In figure 3 we show results for both the quenched (equation [15) and the annealed (equation [16) averages at $T=0.4$ as a function of $q$. We plot data for $N=64,128,256$ and 1024. In order to make the figure readable:

1. we have subtracted from the different measurements a term $1 / 3 \log N$, that separates the points from different system sizes;

2. we have not drawn the $F(q)$ data as points with statistical error bars, but we have drawn a line through the data points.

The (few) data points for $\tilde{F}(q)$ are drawn with statistical error bars (estimated from the variance of sample to sample fluctuations). It appears that the data for $\tilde{F}(q)$ are confined to a much smaller $q$ range than the data for $F(q)$. This is expected since for a given value of $q$, $\tilde{F}(q)$ is well defined only if (the estimate of) $P_{N}(q)$ is larger than zero for every sample, whereas $F(q)$ is well defined as soon as the average $P_{N}(q)$ is larger than zero.

Figure 3 shows that for large system sizes the annealed and quenched estimates are consistent. The same conclusion holds for all the other values of the temperature that we have considered. Because of that we will only consider in what follows the annealed averages $P_{N}(q)$ and $F(q)$. We will estimate statistical errors on our observables by a jack-knife procedure [1].

Our second (and main) numerical goal is to verify the predictions of equation 10 that predict asymptotically a cubic behavior of $1 / N \log P_{N}(q)$ in $q-q_{E A}$, with a coefficient computed at order $\tau$. Now, is all that correct? Can we already observe this behavior on the lattice size that we are able to thermalize in the spin glass phase?

We report our results at different values of the temperature $(T=$ 1.0, 0.8, 0.6 and 0.4) in figures [4, 5 , 6 and 7 respectively. With the dotted line we plot the theoretical prediction of equation [10 With an horizontal arrow we give the analytic value of $P_{N}(q=1)$ (see the discussion later on).

All figures show that results for the systems of different sizes are converging to a common ( $N$ independent) function: our results for the excess free energy are indeed in the asymptotic regime. The careful

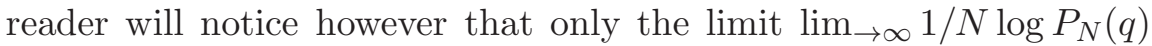
is predicted. There is accordingly an order $1 / N$ ambiguity in the vertical offsets of our plots for $1 / N \log P_{N}(q)$. Plotting for example 


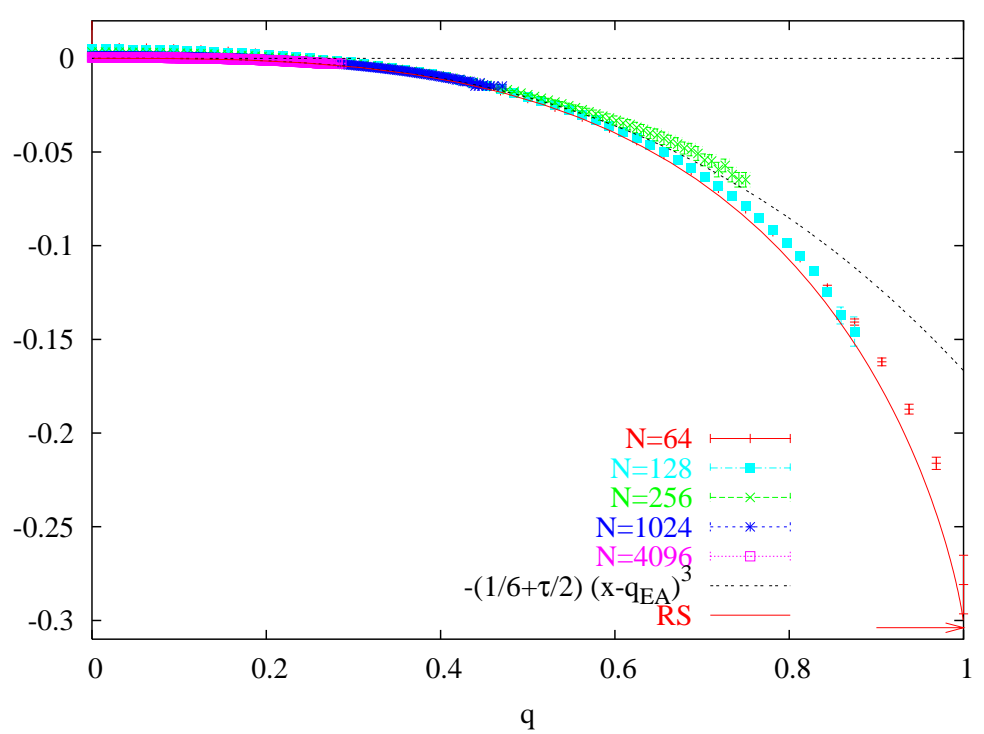

Figure 4: $1 / N \log P_{N}(q)$ versus $q$ for $T=1$ : points with error bars are numerical data from different lattice sizes, the dotted line is from the perturbative estimate in the spin glass phase. An horizontal arrow indicates the exact $q=1$ value. The full line (labeled "RS") is from equation 11.

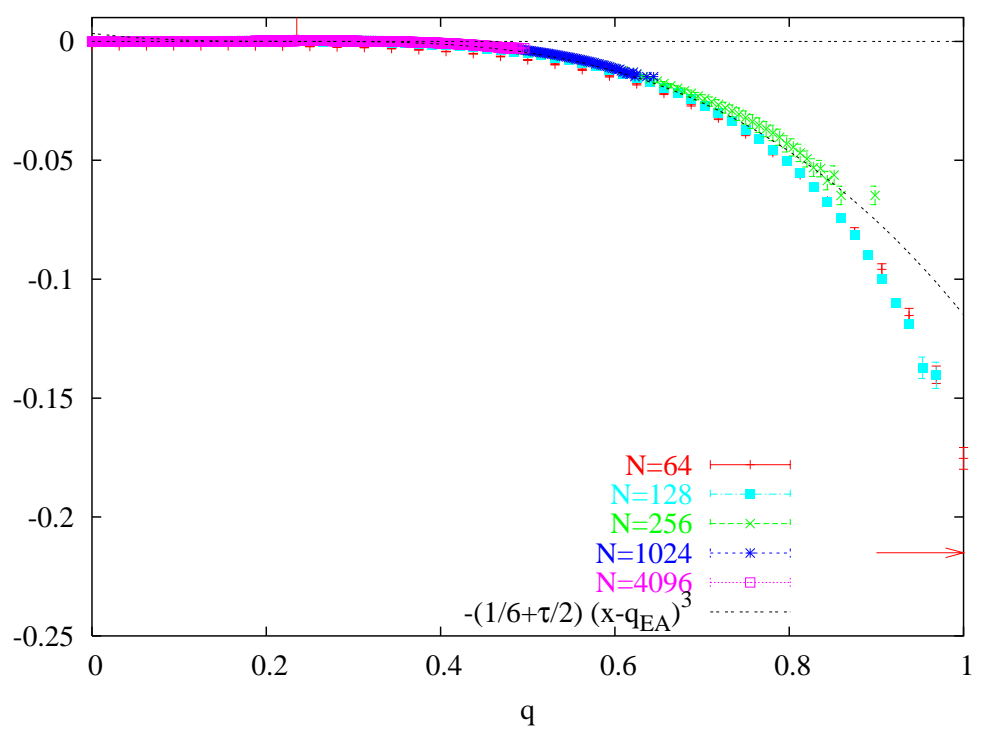

Figure 5: As in figure 4 but with $T=0.8$. The additional vertical arrow indicates the value of $q_{E A}$. 


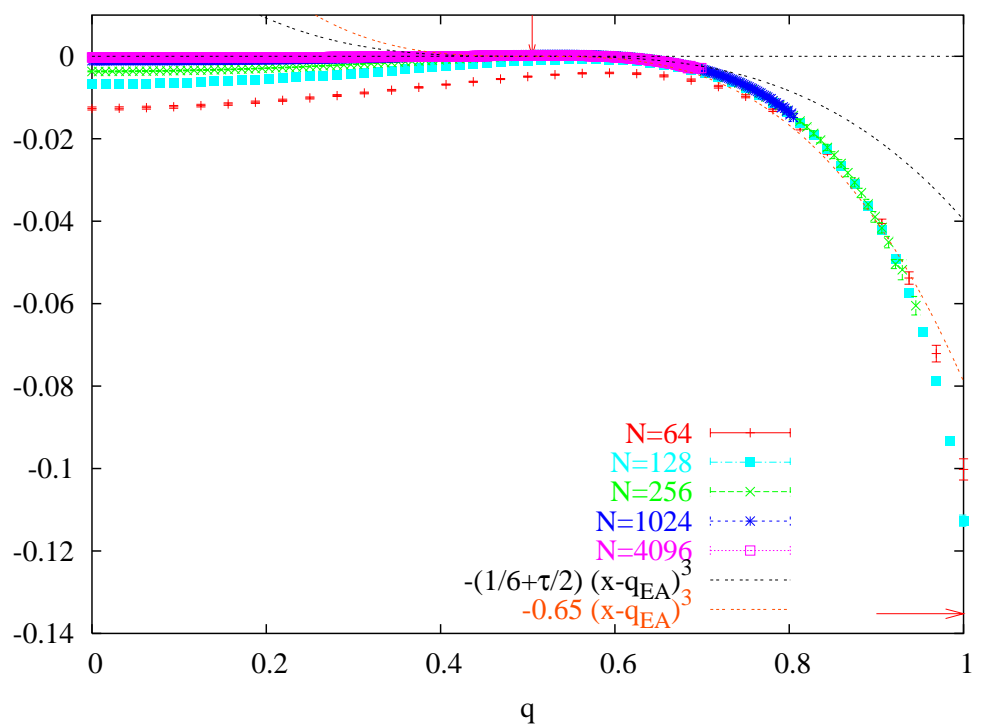

Figure 6: As in figure 5 but with $T=0.6$. The additional lower dotted curve is drawn using a coefficient modified by hand, accounting for the renormalization of the temperature when $\tau$ grows.

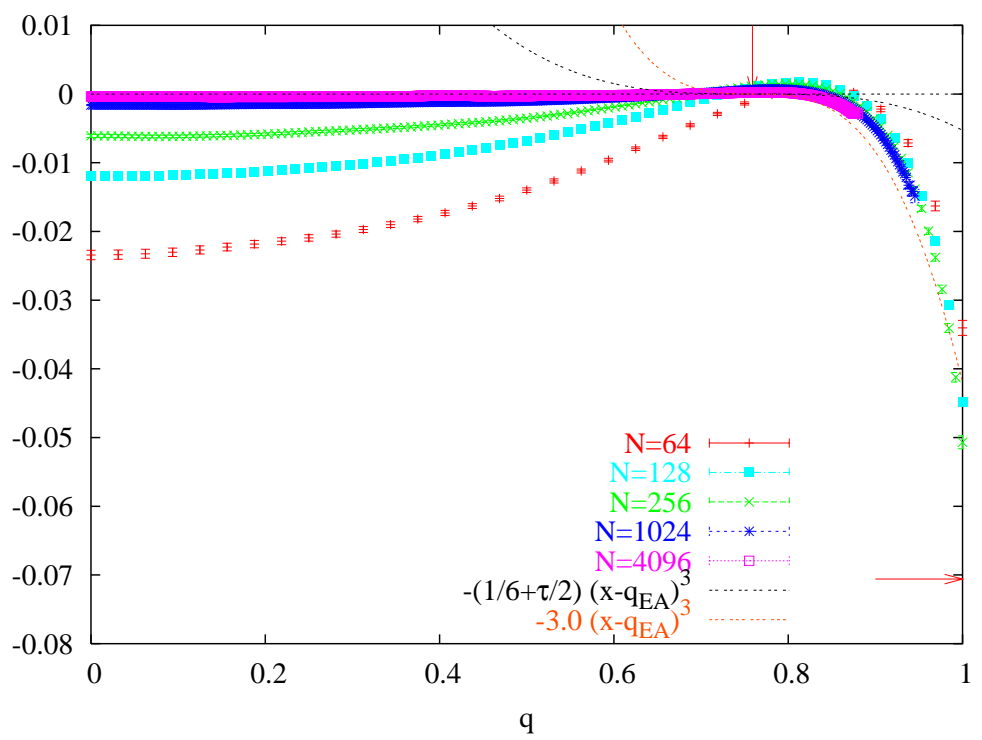

Figure 7: As in figure 6 but with $T=0.4$. 


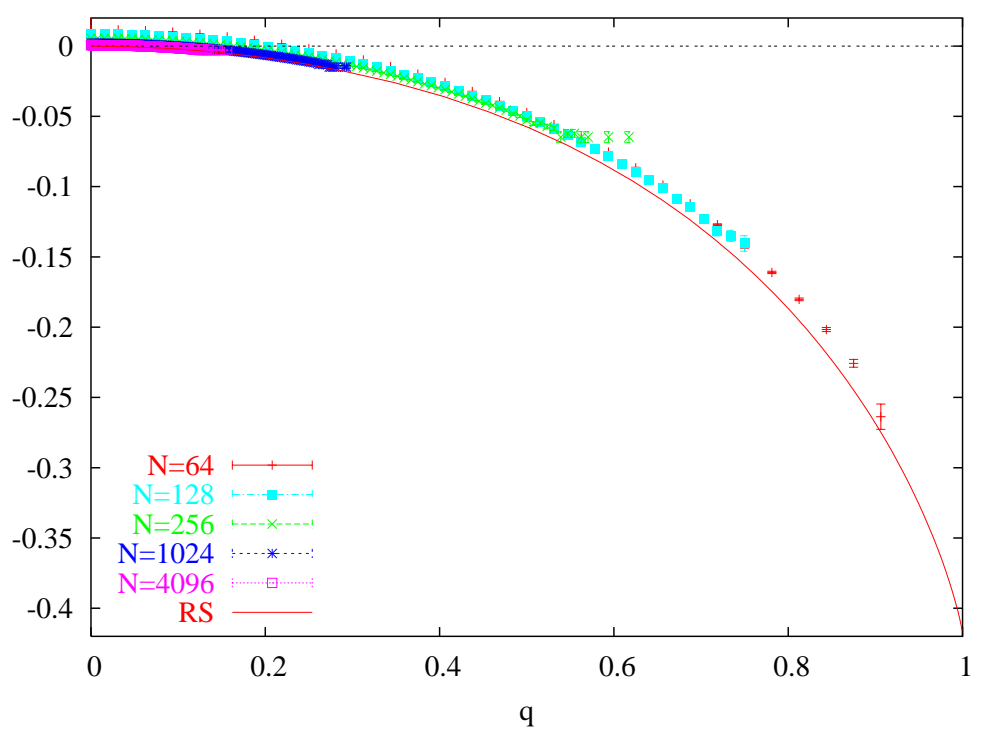

Figure 8: As in figure [ but with $T=1.3$.

$1 / N \log \left(P_{N}(q) N^{-1 / 3}\right)$ would result in a slightly different looking figures.

At $T=1$, where $q_{E A}=0$, the agreement of our data with the theoretical prediction is very good at least up to $q=0.6$. Also at $T=0.8$, where $q_{E A}$ is slightly larger than 0.2 (i.e. still very small) the agreement is very good in a very large $q$ range (up to $q \simeq 0.8$ ). In all these analysis we have used the value of $q_{E A}$ computed by Crisanti and Rizzo by numerical integration [12] of the Parisi solution of the SK model (e.g. we are not using $q_{E A}$ computed to order $\tau$ ).

At $T=0.6$, where $q_{E A}$ is of order 0.5 and a small $\tau$ expansion with two terms starts to be inappropriate, the agreement starts to be less good. To take into account the fact that here $\tau$ is large and there is an effective renormalization (when $T=0.6$ the first order correction in equation 10 is equal to 0.16 while the leading term is equal to $1 / 6$ ) we have added a further curve where we substitute the prefactor we have computed analytically in equation 10 with some effective value, that optimizes the matching of the analytic form to the numerical data: this improves very much the agreement. This effect is even more dramatic at $T=0.4$, where again a cubic dependence with a renormalized prefactor fits very well the data in a large $q>q_{E A}$ range. 
Our third numerical goal concerns the value of of $P_{N}(q)$ at $q=1$ (that we plot with an horizontal arrow in the four figures). We use the simple relation

$$
\frac{1}{N} \log P_{N}(q=1)=-\frac{2}{T}\left(\mathcal{F}_{\frac{T}{2}}-\mathcal{F}_{T}\right),
$$

between $P_{N}(q=1)$ at temperature $T$ and the free energy of the SK model at temperature $T$ and $T / 2$. We take for $\mathcal{F}_{T}$ the infinite volume free energy of the model derived with high precision in [12] from Parisi solution.

One sees from figures 4.7 that the prediction of equation 17 for $P_{N}(q=1)$ is smaller than the value that formula [10 takes at $q=1$ : obviously this had to be expected, since equation 10 is the first term in an expansion in powers of $q-q_{E A}$, and is not supposed to be valid up to the $q=1$ boundary, where higher orders cannot be neglected (the function is presumably singular at $q=1$ ). Note however that the interplay between finite size effects and higher orders in $T_{c}-T$ is not trivial. It could be studied numerically using the multi-overlap algorithm [13] which allows to measure $P_{N}(q)$ with high accuracy up to $q=1$. However parallel tempering numerical data, when pushed to very high statistics, confirm already in a remarkable way the analytic value (this can be only done for small lattice sizes). At $T=1$ we are able to push the $N=64$ lattice up to $q=1$ and to keep under control the limit of the $N=128$ lattice. At lower $T$ values the check is slightly more difficult, but one sees that the $q=1$ limit of larger and larger lattices approaches better and better the analytic value.

That the situation is well under control can be shown nicely for $T \geq 1$ by comparing the numerical data, in the whole $q \in[0,1]$ range, with the results of our RS computation of equation [1] This is done in figures 4 for $T=1.0$ and 8 for $T=1.3$ (the highest temperature alas in our parallel tempering simulation). The first figure shows the full line (labeled "RS") cleanly overshooting the dotted line, to reach the $q=1$ axis slightly above (as expected) the exact $q=1$ limit $^{3}$. Figure 8 show also again the agreement our our numerics with the replica calculation.

Equation [10 suggests [7, 8] that (for $T<T_{c}$ ) $P_{N}(q)$ scales asymptotically as

$$
P_{N}(q) N^{-\frac{1}{3}}=\mathcal{F}\left(N\left(q-q_{E A}\right)^{3}\right) \quad \text { for } q>q_{E A},
$$

\footnotetext{
${ }^{3}$ This strongly indicates that the correct large $q$ behavior in figures 4 and 15 is given by the $N=64$ and 128 data, and that the rightmost $N=256$ points are somewhat misleading
} 


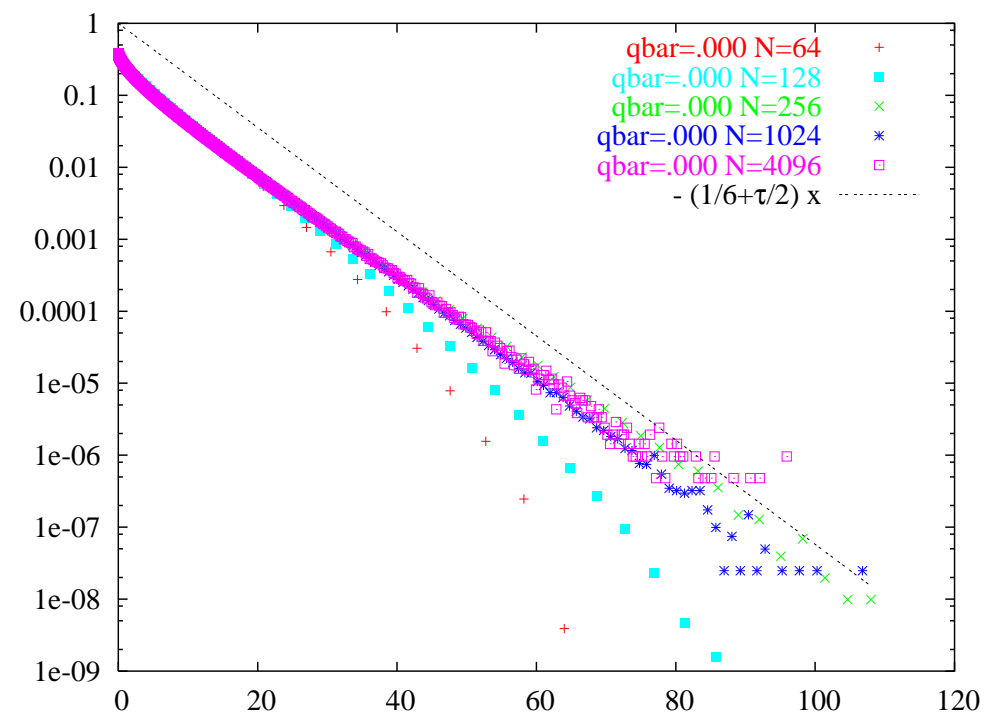

Figure 9: Scaling plot of $P_{N}(q) N^{-\frac{1}{3}}$ as a function of $\left(q-q_{\max }(N)\right)^{3} N$ for $T=1.0$, compared with the coupled replica estimate (with arbitrary normalization). Our estimate of $q_{\max }(N)$ is reported in the figure under the name qbar (it is obviously zero at $T_{c}$ ). 


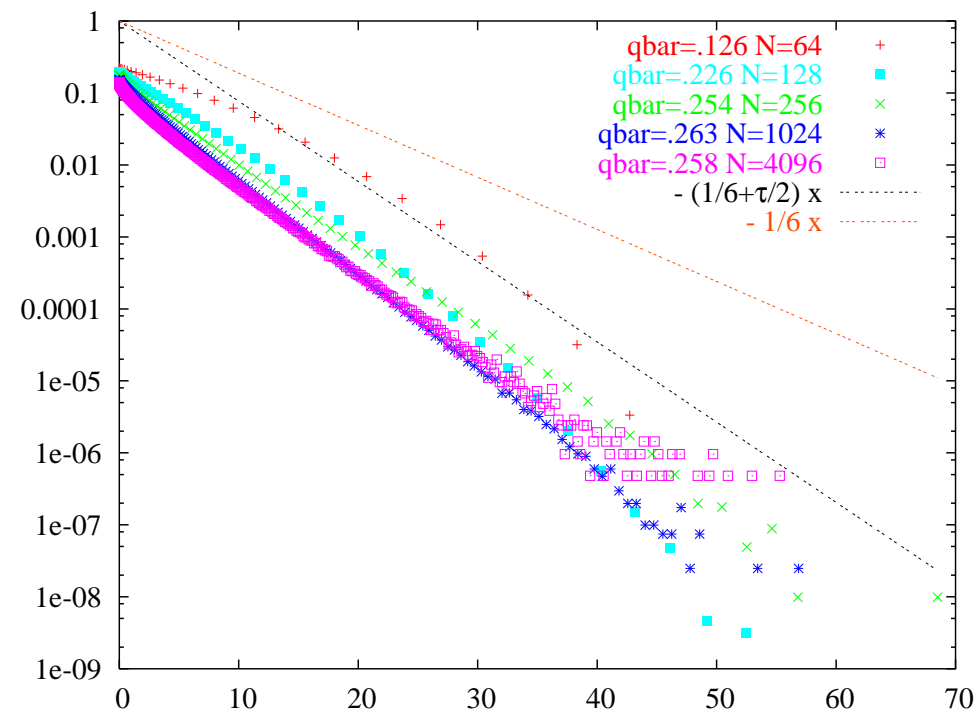

Figure 10: As in figure 9] but for $T=0.8$. Coupled replica estimate is plotted with and without the term linear in $\tau$. Note that $q_{\max }(N)$ is not well determined on small systems at this temperature, that is close to $T_{c}$.

with $\mathcal{F}(z) \propto \exp (-A z)$, with some positive constant $A$, for large $z$. If the above formula holds down to the maximum of $P_{N}(q)$ this implies that the location of the peak of the probability distribution of the overlap on a lattice of size $N$, that we denote by $q_{\max }(N)$, has to behave like

$$
q_{\max }(N)=q_{E A}+c N^{-\frac{1}{3}},
$$

a behavior that has indeed been verified with high accuracy in former work [5].

In what follows we will accordingly look for a scaling law in the form

$$
P_{N}(q) N^{-\frac{1}{3}}=\mathcal{G}\left(N\left(q-q_{\max (N)}\right)^{3}\right)
$$

using the numerical estimates obtained for $q_{\max (N)}$. Figures 9,10111 and 12 are scaling plots of $\log \left(P_{N}(q) N^{-1 / 3}\right)$ versus $N\left(q-q_{\max }(N)\right)^{3}$. The figures clearly exhibit the predicted cubic limiting behavior. At $T=T_{c}$ the data are furthermore in excellent agreement with the predicted slope. At $T=0.8$ the term linear in $\tau$ computed in this paper is essential in order to obtain good agreement with the data. $T=0.6$ and $T=0.4$ are clearly too far from $T_{c}$ in order to obtain 


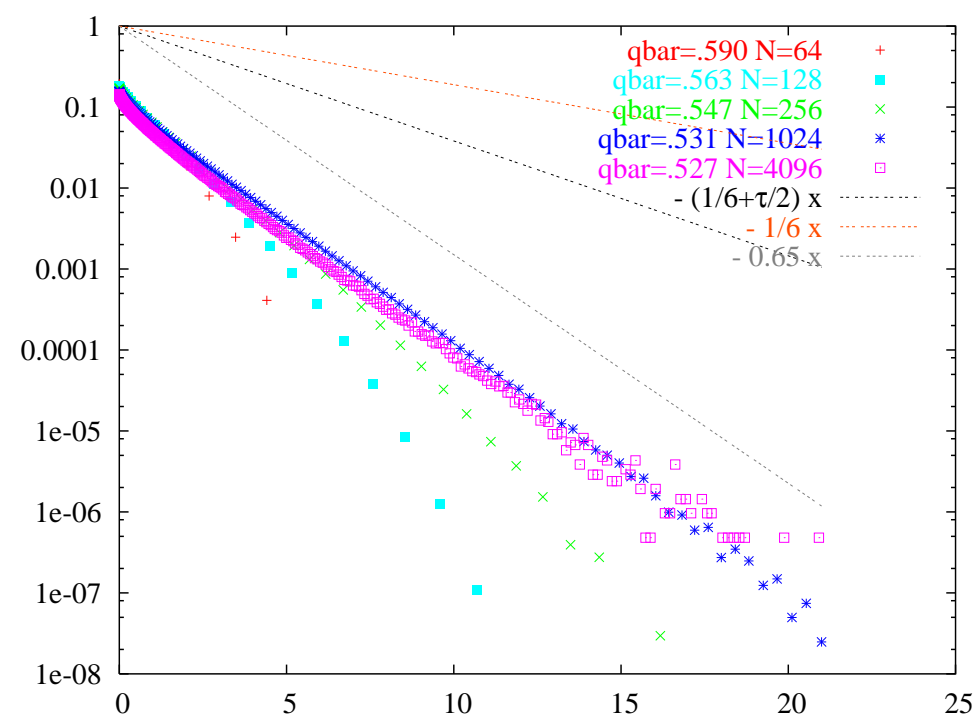

Figure 11: As in figure 10 but for $T=0.6$. The additional dotted line is drawn using the same hand modified coefficient as in figure [6.

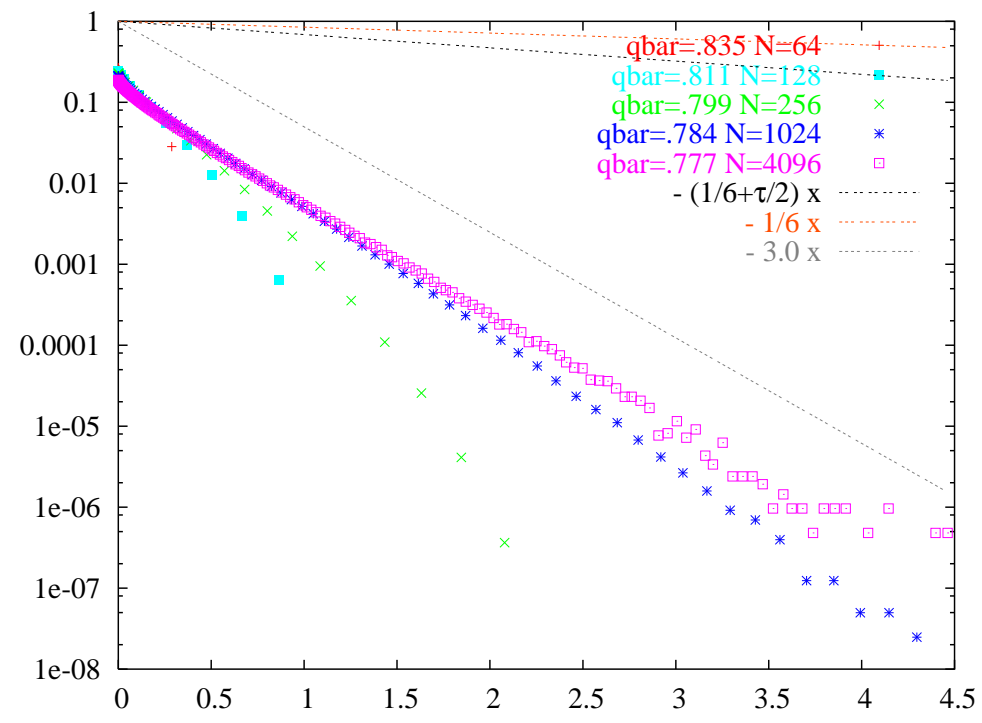

Figure 12: As in figure 9 but for $T=0.4$. The additional dotted line is drawn using the same hand modified coefficient as in figure $\mathbf{Z}$ 
a good result for the slope with only two terms in the expansion in powers of $\tau$. Scaling is violated on small systems for the rightmost points. It must be so since those are points with $q$ close to one, and we have seen before that close to one the behavior is not cubic.

It should be stressed that with our data scaling in $\left(q-q_{\max }(N)\right)^{3} N$ (equation 18) is much better than scaling in $\left(q-q_{E A}\right)^{3} N$ (equation 19] using the very precise data for $q_{E A}$ by Crisanti and Rizzo). One reason for this better agreement may be that for $q>q_{E A}$ the inflection point of $\left(q-q_{\max (N)}\right)^{3} N$ mimics the maximum of $1 / N \log P_{N}(q)$. Scaling plots as function of $\left(q-q_{E A}\right)^{3} N$ with an ad-hoc effective $N$ independent $q_{E A}$ appear (in a small $z$ interval) in [7] for $T=0.8$, and in [8] for $T=0.5$.

\section{Conclusions}

The results of these analysis are very positive. We have first argued that the large deviation leading behavior of $P_{N}\left(q>q_{E A}\right)$ is generically $1 / N \log P_{N}(q)=-\mathcal{A}\left(q-q_{E A}\right)^{3}$, and we have computed the first corrections to the expansion of $\mathcal{A}$ in powers of $T_{c}-T$ for the Sherrington-Kirkpatrick model.

Data from numerical simulations confirm that this behavior can be detected already on moderate lattice sizes. First we have seen that we are well allowed to take an annealed average of $F$. Secondly we have analyzed the details of the cubic behavior, and checked the validity of the perturbative estimate of the prefactor: close to $T_{c}$ it works very well, while far away from $T_{c}$ a simple renormalization improves the agreement in a substantial way. Our very accurate data have allowed a detailed analysis of the $P(q=1)$ value, allowing again for a positive check of the analytic result. For $T \geq T_{c}$ an exact computation in the replica symmetric scheme shows nicely the crossover from the $q \approx q_{E A}$ behavior to the ultimate limit $q=1$. At last we have shown that the scaling plots for $P_{N}(q)$ improve appreciably if one uses $\left(q-q_{\max }(N)\right)^{3} N$ as the scaling variable, showing clearly the predicted cubic behavior of $\log \left(P_{N}(q) N^{-1 / 3}\right)$. 


\section{Acknowledgments}

We thank A. Crisanti and T. Rizzo for providing us with their unpublished numerical evaluations of quantities in the SK model, and Giorgio Parisi for conversations. SF thanks the SPhT Saclay for a visiting professor fellowship and warm hospitality in the months of OctoberDecember 2001, during which part of this work was performed.

\section{References}

[1] G. Parisi, Phys. Rev. Lett. 43 (1979) 1754.

[2] M. Mézard, G. Parisi, and M.A. Virasoro, Spin Glass Theory and Beyond (World Scientific, Singapore, 1987).

[3] N. D. MacKenzie and P. Young, Phys. Rev. Lett. 49 (1982) 301;

A. Billoire and E. Marinari, J. Phys. A 34 (2001) L1.

[4] See for example A. Billoire and E. Marinari, J. Phys. A 33 (2000) L265, and references therein.

[5] A. Billoire and E. Marinari, preprint cond-mat/0202473, to appear in Europhysics Letters.

[6] S. Franz, G. Parisi and M. A. Virasoro, J. Phys. I (France) 2 (1992) 1869.

[7] G. Parisi, F. Ritort and F. Slanina, J. Phys. A 26 (1993) 3775.

[8] J.C. Ciria, G. Parisi, and F. Ritort, J. Phys. A 26 (1993) 6731.

[9] S. Franz, PhD Thesis, unpublished.

[10] For an introduction see for example E. Marinari, in Advances in Computer Simulations, edited by J. Kerstez and I. Kondor (Springer-Verlag, Berlin 1998), p.50, preprint cond-mat/9612010

[11] H. Flyvbjerg, in the same book of reference [10.

[12] A. Crisanti and T. Rizzo, Phys. Rev. E 65, 046137 (2002), preprint cond-mat/0111037, and private communication.

[13] B. Berg, A. Billoire and W. Janke, Phys. Rev. E 65 (2002) 045102R, preprint cond-mat/0108034 preprint cond-mat/0205377 to appear in Phys.Rev. E. 